\title{
Novel Gap Filling BARC with High Chemical Resistance
}

\author{
Yuto Hashimoto*, Shigetaka Otagiri, Hiroto Ogata, Satoshi Kamibayashi, \\ Ryuta Mizuochi, Takahumi Endo, Yuki Endo, and Takahiro Kishioka
}

\author{
Semiconductor Materials Research Department, Materials Research Laboratories, \\ Nissan Chemical Industries, Ltd., 635 Sasakura, Fuchu-machi, Toyama 939-2792, Japan \\ *yhashimoto@nissanchem.co.jp
}

\begin{abstract}
In the recent of the semiconductor manufacturing process, variety of properties (narrow gap-filling and planarity etc.) are required to organic BARC in addition to the conventional requirements. Moreover, $\mathrm{SC}-1$ resistance is also needed because BARC is often used as a wet etching mask when TiN processing. But conventional BARC which include crosslinker does not have enough SC-1 resistance, and we found that it is also difficult to obtain good gapfilling and good planarity because of outgassing and film shrinkage derived from the crosslinker. In this study, we have developed the new self-crosslinking BARC. The new crosslinking system shows low outgassing and film shrinkage because of not including crosslinker. So, novel BARC has better gap filling property and planarity and over 3 times higher SC-1 resistance than that of conventional BARC. Moreover, by adding the low molecular weight additive which has high adhesive unit to TiN surface, the novel BARC has over 10 times higher SC-1 resistance than that of conventional BARC. And this novel BARC can be applied both $\mathrm{ArF} \& \mathrm{KrF}$ lithography process because of broad absorbance, high etching rate, chemical resistance (SC-1, SC-2, DHF, and others) and good film thickness uniformity. In this paper, we will discuss the detail of new self-crosslinking BARC in excellent total performance and our approach to achieve high chemical resistance.
\end{abstract}

Keywords: BARC, Gap filling, Planarity, Chemical resistance, Self-crosslinking, ArF, KrF

\section{Introduction}

The Bottom Anti-Reflective Coating (BARC) material is the key technology for optical lithography (i-line, $\mathrm{KrF}, \mathrm{ArF}$ and $\mathrm{ArFi}$ ) to prevent reflection issue between $\mathrm{PR}$ and substrate interface. Much is required of the BARC, such as improved process margin, control of optical parameters, high etch rate, defect free and broad compatibility with photoresist [1,2].

The lithography process for implant layer will be as a critical layer to manufacture process because of the CD shrinkage the tighter pitch and higher topography beyond $7 \mathrm{~nm}$ node. Because of this, variety of properties (narrow gap-filling and planarity on topography pattern) are required to organic BARC in addition to the conventional requirements.

When BARC is coated on topography pattern, the void which is gap filling fail issue in narrow space often becomes problem in the semiconductor manufacturing process. The void is occurred the deterioration of device properties, because of etching damage to substrate that it depends on etching nonuniformity. In particular, gap size is under $10 \mathrm{~nm}$ beyond $7 \mathrm{~nm}$ node, it is difficult to achieve the required properties. Achieving this requirement is key to improve property of the advanced devices.

On the other hand, other important property is required for BARC. SC-1 resistance is also needed because BARC is often used as a wet etching mask when TiN processing in the recent High-K/Metal Gate process (HKMG). In case of using poor chemical resistance BARC, throughput is extremely deteriorated because it is necessary to change the chemical composition and carry out the treatment for a long time under weak conditions. To improve the process throughput, high chemical resistance 
BARC is needed.

Conventional BARC which include crosslinker doesn't have enough SC-1 resistance, and we found that it is also difficult to obtain good gap-filling and good planarity because of outgassing and film shrinkage derived from the crosslinker. In this study, we have developed the new self-crosslinking BARC with new crosslinking system to achieve above properties. In this paper, we will discuss the detail of new self-crosslinking BARC in excellent total performance and our approach to achieve high chemical resistance.

\section{Experimental}

2.1. Preparation of materials

All samples were the thermal crosslink material and basically including base polymer and some additives for Crosslinking reaction. Propylene glycol mono methyl ether (PGME), Propylene glycol mono methyl ether acetate (PGMEA), etc. are used as solvent system.

The BARC film was prepared under $1500 \mathrm{rpm} / 60$ sec. and $250{ }^{\circ} \mathrm{C} / 60 \mathrm{sec}$ bake condition. The formulated materials were filtrated with $0.01 \mu \mathrm{m}$ UPE filter. Optical interference film thickness measurement tool (Nanospec AFT6100, Nanometrics) was used for thickness measurement.

\subsection{IR measurement}

The BARC coated on Cr substrate at several bake temperature. IR measurement was evaluated by using the tool and condition as shown below:

IR measurement tool: NicoletiS50 (Thermo),

Measurement method: Reflection method.

\subsection{Stripping test}

In some of the following examples, a stripping test was performed to determine the resistances of the experimental BARC to photoresist solvents. In this procedure, the BARC were spin-coated on a silicon wafer and baked. The thickness of the BARC was then measured. PGME/PGMEA as photoresist solvent was dispensed on the silicon wafer on which BARC was coated, then kept for $60 \mathrm{~s}$, followed by spin drying at $3500 \mathrm{rpm}$ for $30 \mathrm{~s}$ to remove the solvent. The film was then baked on a hot plate at $100{ }^{\circ} \mathrm{C}$ for $30 \mathrm{~s}$. The thickness of the BARC was again measured. The amount of stripping was determined to be the difference between the initial and final thicknesses. A stripping test result of less than $2 \mathrm{~nm}$ was considered acceptable to avoid resist mixing issue for lithography.

\subsection{Gap filling}

The gap filling property on topography substrate was evaluated by using narrow trench substrate (space $10 \mathrm{~nm}$, depth $230 \mathrm{~nm}$ ) made with $\mathrm{SiO}_{2}$ substrate. BARC was coated on this topography substrate and gap filling check was evaluated by using cross-section SEM (Hitachi S4800).

\subsection{Chemical resistance}

BARC was coated on TiN wafer $(3 \mathrm{~cm} \times 3 \mathrm{~cm})$ and baked on a hotplate. The coated wafer was soaked in $\mathrm{SC}-1$ solution heated to $50{ }^{\circ} \mathrm{C}$. $\mathrm{SC}-1$ resistance was measured film peeling time from TiN substrate. The $\mathrm{SC}-1$ condition was shown below:

SC-1 solution: 30 vol $\% \mathrm{NH}_{3}$ aq $/ 30$ vol $\% \mathrm{H}_{2} \mathrm{O}_{2}$ aq/ DIW $=1: 1: 2$.

\subsection{Etching rate}

The BARC was spin-coated on a silicon wafer and baked on a hotplate. Then the BARC film was etched by $\mathrm{N}_{2}$ gas. The Etching condition was shown below:

Tool: RIE-10NR (SAMCO),

Gas: $\mathrm{N}_{2}$, Press: $15 \mathrm{~Pa}$, Temperature: $23{ }^{\circ} \mathrm{C}$, Flow rate: $200 \mathrm{sccm}$, Time: $1 \mathrm{~min}$., RF power: $100 \mathrm{~W}$.

The etch rate is calculated by differentiation of film thickness at before etching and after etching is divided by the etching time.

\subsection{Measurement of the optical parameters}

The optical parameters ( $n$ value and $k$ value) of the BARC films were measured by the VUV-VASE32 ellipsometer (J. A. Woollam Co., Inc.).

\subsection{Lithography}

BARC was spin-coated at $200 \mathrm{~nm}$ and baked at $250{ }^{\circ} \mathrm{C}$ for $60 \mathrm{sec}$. The appropriate resist was coated on the BARC. Lithographic test of exposure light compatibility was evaluated by using below tool and resist:

$\mathrm{KrF}$ lithography: tool (0.75NA: half annular $\sigma$ 0.85/0.43), resist KrF PR A,

ArF lithography: tool (0.9NA: $2 / 3$ annular $\sigma$ 0.93/0.62), resist ArF PR A,

ArFi lithography (imec): tool (1.35NA: Dipole40Y $\sigma 0.98 / 0.81 / \mathrm{X})$, resist ArFi PR A.

The resist was coated by the recommended conditions and was developed using TMAH developer. The pattern profiles were observed by cross-sections SEM.

\section{Results and discussion}

3.1. Material design and fundamental study 


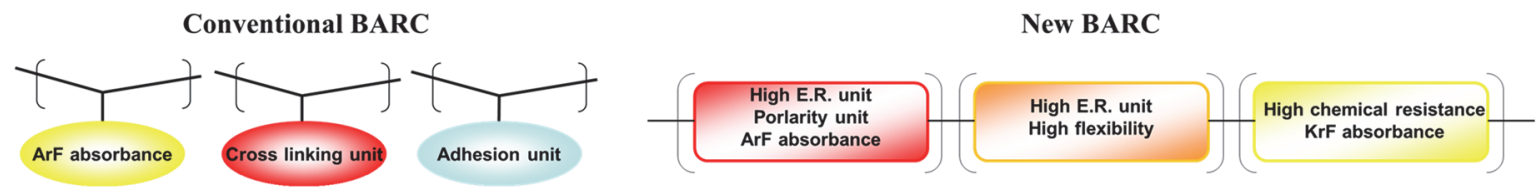

Fig.1. Base polymer design of new gap filling BARC.

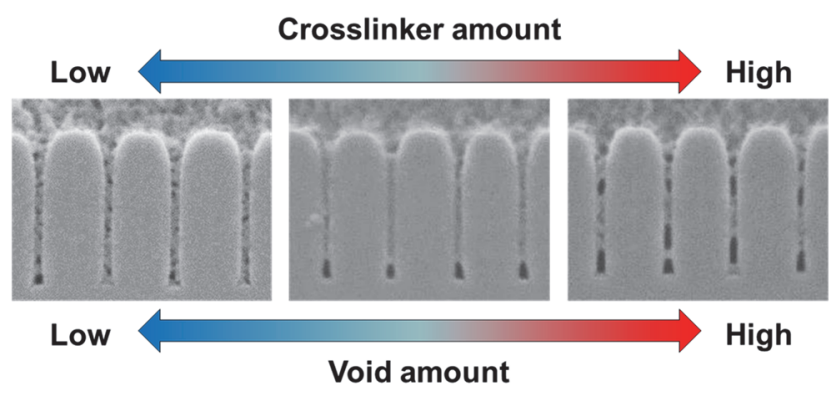

Fig.2. Crosslinker amount dependency of gap filling property.

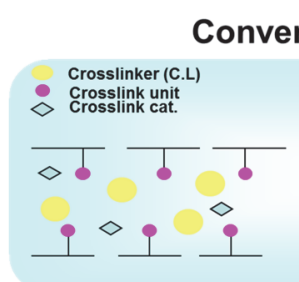

Conventional BARC

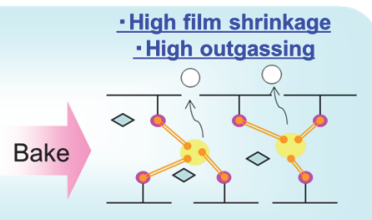

Fig.3. BARC crosslinking system image.
New BARC (Self cross-linking system)

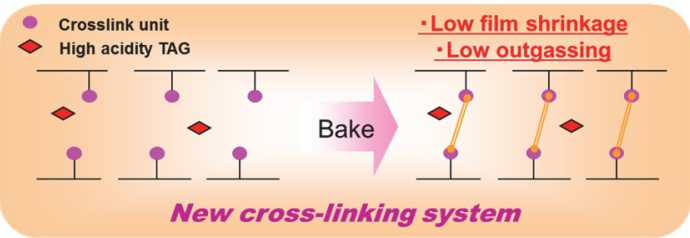

\subsubsection{Material design of new BARC}

In the advanced process, BARC is not only needed good lithographic performance, but also the following properties are required.

1. Good gap filling property in narrow trench $(<10 \mathrm{~nm}$ gap).

2. Good chemical resistance (SC-1, SC-2, and others).

3. Good lithographic performance at broadband exposure tool ( $\mathrm{KrF}, \mathrm{ArF}, \mathrm{ArFi})$.

4. High E.R.

For these requirements, we designed the base polymer structure as follow (Fig.1).

The new BARC was selected polyester type material to get high E.R. The polarity unit aim to be the adhesive unit between BARC and PR for pattern collapse margin, the flexibility unit is for good gap filling property by reducing polymer $\mathrm{Tg}$ and adding high fluidity. New BARC can be used at various exposure process because of including $\mathrm{ArF}$ chromophore and $\mathrm{KrF}$ chromophore. And, this $\mathrm{KrF}$ chromophore is also introduced to improve the chemical resistance. Moreover, it is easy to control E.R. and optical parameter by adjusting the introduction ratio of each unit.

\subsubsection{Analysis of new cross-linking system}

The gap filling property in narrow gap is due to various properties such as polymer $T \mathrm{~g}$, polymer size, reflow property, viscosity, outgassing, film shrinkage, and so on $[3,4]$. Especially, conventional BARC is formed from main polymer, cross-linker and catalyst, this cross-linking system affect bad gap filling property because of outgassing and film shrinkage derived from the crosslinker. Figure 2 was shown relationship between gap filling property and amount of crosslinker in conventional BARC. It is found that the void amount increases in proportion to crosslinker amount. Therefore, we tried further improvement of gap filling property to develop the new self-crosslinking BARC not using conventional cross linking system. Figure 3 was shown the model image of new self-crosslinking system.

This crosslinking reaction is characterized by bonding of polymers to each other. But, it is difficult to be applied in BARC formed process because this crosslinking reaction is needed high-activated catalyst and high temperature baking process. However, we found the key catalyst which enables this crosslinking reaction in low temperature baking process. Figure 4 was shown the crosslinking reaction speed of BARC which used the new catalyst by using IR measurement and stripping test. This crosslinking reaction start to decrease the crosslinking unit from $130{ }^{\circ} \mathrm{C}$, about $80 \%$ crosslinking unit is consumed over $215{ }^{\circ} \mathrm{C}$. This 


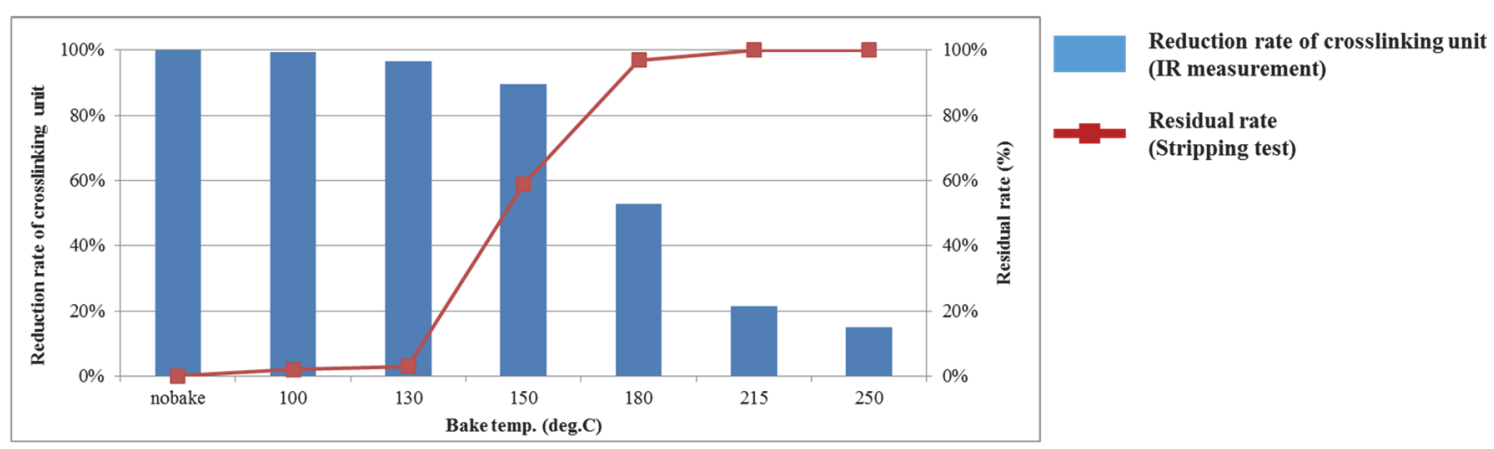

Fig.4. Reaction speed analysis of new self-crosslinking system.

crosslinking system could be applied in BARC formed process because solvent resistance was obtained over $215^{\circ} \mathrm{C}$.

\subsubsection{Improvement of chemical resistance}

In the recent of the semiconductor manufacturing process, BARC is needed various chemical resistance as the important property. Especially, SC1 solution is used for wet etching process to TiN layer, next generation BARC should be show high $\mathrm{SC}-1$ resistance. Figure 5 shows the process flow during TiN processing. Through this process, Penetration of SC-1 solution mainly occurs at BARC/TiN interface. Almost all problems are BARC peeling from TiN surface. To get high SC-1 resistance, it is particularly important to improve the adhesion of the BARC / TiN interface.

BARC with conventional crosslinking system shows poor SC-1 resistance which is low adhesion to TiN. But, the new self-crosslinking BARC shows 3 times higher SC-1 resistance than that of conventional BARC. It was found from the result that the conventional crosslinker deteriorates the adhesion to TiN. Since there is a different in properties with or without crosslinker, it is expected that adhesion of BARC/TiN interface can be controlled by adding additives to BARC. Through the screening of additives, we found one characteristic additives. This additive has bulky backbone and high adhesion unit to TiN surface. The BARC showed excellent SC-1 resistance which was introduced new additive. To confirm the effect of new additive, we evaluated additive amount dependency of SC-1 resistance (Fig. 6). Control sample is self-crosslinking BARC without additive. By increasing the additive amount, it was confirmed improvement of SC-1 resistance. But, The SC-1 resistance tended to be saturated with additive above a certain level. Regarding this phenomenon, it was expected that additive was gradually coordinated on TiN surface with increasing additive amount and TiN surface was saturated with additive above a certain level. The model image of this phenomenon was shown below (Fig. 7).

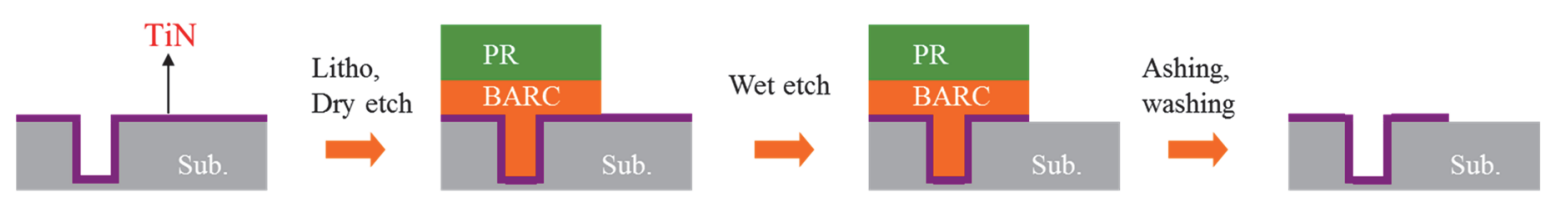

Fig 5. The process flow during TiN processing.

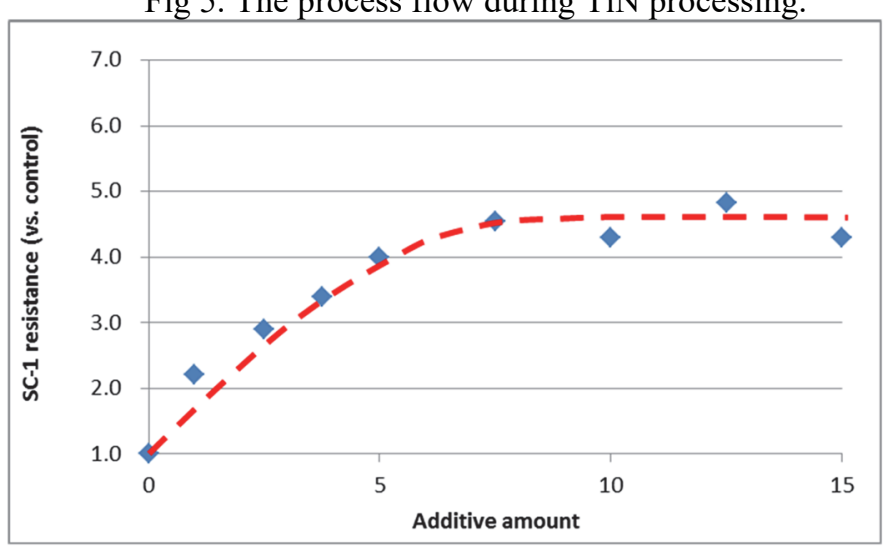

Fig.6. Additive amount dependency of SC-1 resistance. 


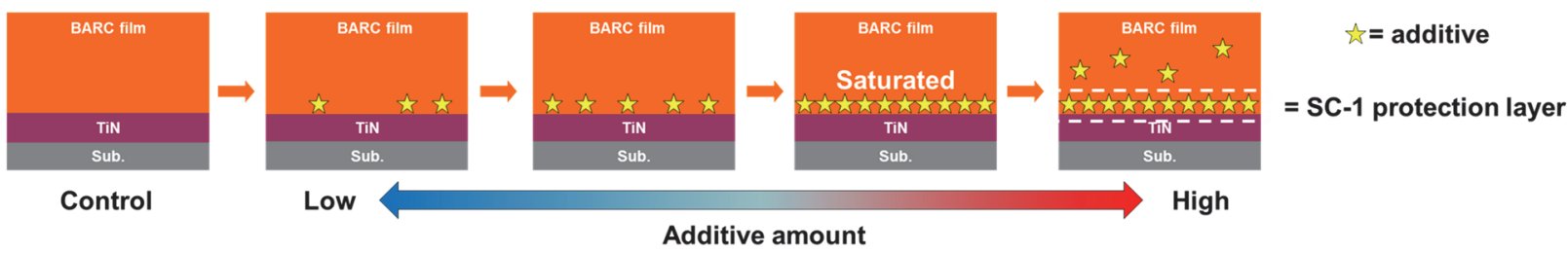

Fig.7. Model image of additive effect.

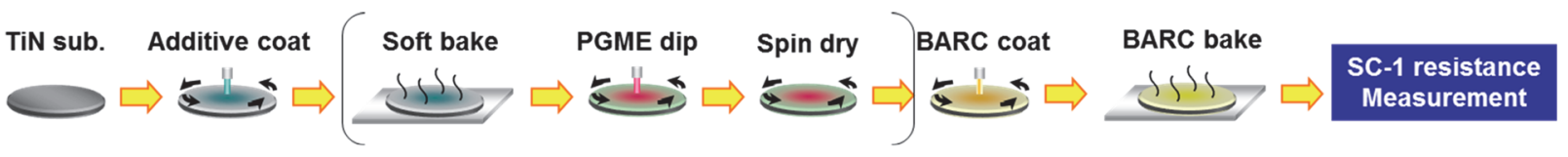

\begin{tabular}{|c|c|c|c|c|c|}
\hline Process & Additive coating & Additive soft bake & Additive stripping & BARC coating & BARC baking \\
\hline Control & w/o & w/o & w/o & w & w \\
\hline Process A & w & w/o & w/o & w & w \\
\hline Process B & w & w/o & w & w & w \\
\hline Process C & w & w & w/o & w & w \\
\hline Process D & w & w & w & w & w \\
\hline
\end{tabular}

Fig.8. Experimental flow and process conditions.

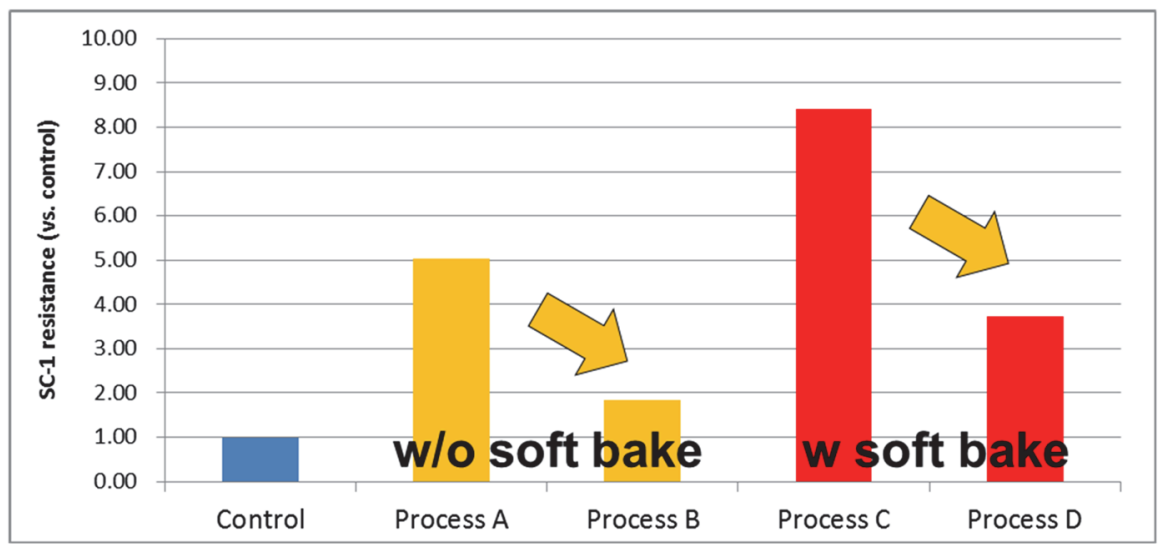

Fig.9. Additive effect as adhesion agent in various processes.

Next, in order to clarify the proof of this theory, it was verified whether the same effect can be obtained by using additive as adhesion agent. Moreover, we verified in various processes when additive was coordinated on TiN surface (Fig. 8). Additive solution was prepared as $10 \%$ PGME solution. Self-crosslinking BARC without this additive was used as control sample and upper layer. Figure 9 was shown results of this verification experiment.

In all processes, we confirmed the improvement of $\mathrm{SC}-1$ resistance. Since process B and D showed lower $\mathrm{SC}-1$ resistance than that of process $\mathrm{A}$ and $\mathrm{B}$, it became clear that additive dose not react with TiN surface by chemical bond.

Moreover, we found that additive was more coordinated through the bake process because process $\mathrm{C}$ and $\mathrm{D}$ showed higher SC-1 resistance than that of process $\mathrm{A}$ and $\mathrm{B}$.

From above results, it was proved that we can control the SC-1 resistance to use new additive with high adhesion unit to TiN surface.

\subsection{Performance of optimized new BARC}

3.2.1. Gap filling performance

NCA42219 is the new self-crosslinking gap filling BARC which was optimized acid catalyst amount and additive amount to achieve the various required properties. We evaluated gap filling property of NCA42219. Control sample is conventional gap filling BARC with crosslinker. Regarding NCA42219, the void was not observed in narrow trench pattern (Fig. 10).

\subsubsection{SC-1 resistance}

We evaluated $\mathrm{SC}-1$ resistance of the new self- 

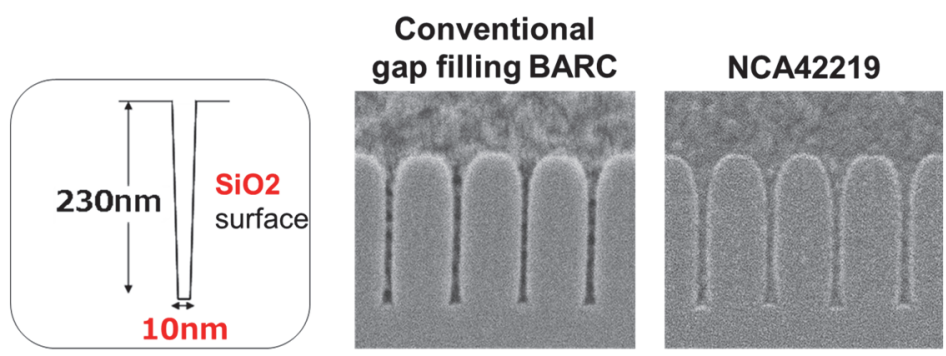

Fig. 10. Gap filling property of NCA42219.

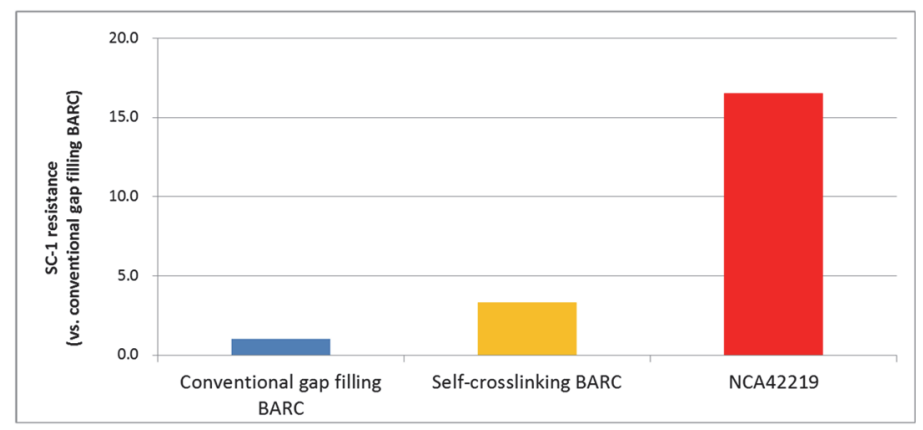

Fig.11. SC-1 resistance of NCA42219.

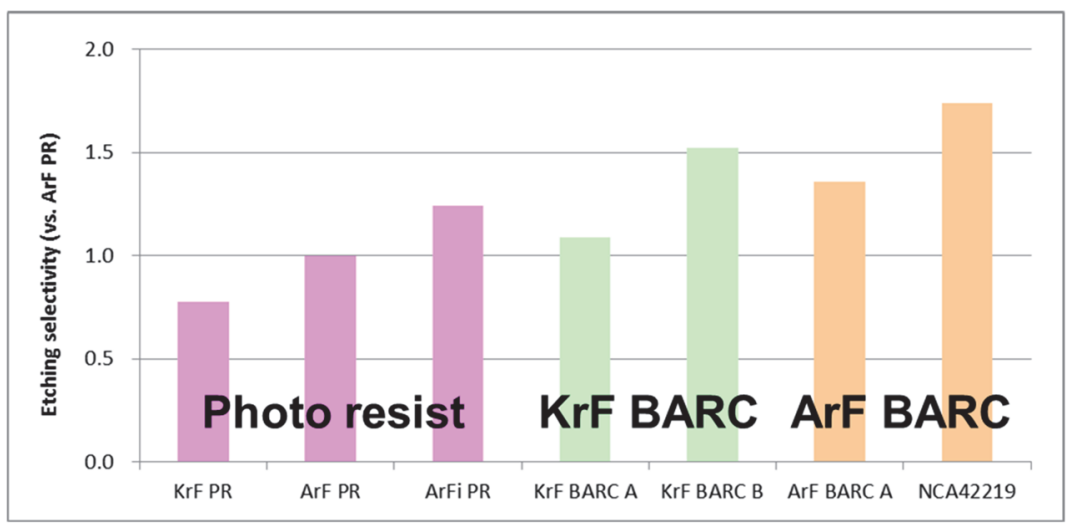

Fig.12. Etching selectivity of NCA42219.

\begin{tabular}{|c|c|c|c|c|}
\hline BARC $n / k$ & & $\mathrm{n} / \mathrm{k} 193 \mathrm{~nm}: 1.82$ & $8 \mathrm{~nm}: 1.74 / 0.14$ & \\
\hline Target pattern & L170P340 & L100P200 & L50P100 & L50P100 \\
\hline \multirow{4}{*}{ Stack } & & & PR (105nm) & PR (105nm) \\
\hline & PR (550nm) & PR (200nm) & BARC (200nm) & $\operatorname{BARC}(28 \mathrm{~nm})$ \\
\hline & BARC (200nm) & BARC $(200 \mathrm{~nm})$ & SiON (50nm) & SION (50nm) \\
\hline & Bare-Si & Bare-Si & Bare-Si & Bare-Si \\
\hline Reflectivity (\%) & 1.0 & 0.5 & 0.9 & 0.2 \\
\hline $\operatorname{EOP}\left(\mathrm{mJ} / \mathrm{cm}^{2}\right)$ & 29 & 29 & 24 & 23 \\
\hline $\mathrm{DOF} ; \mathrm{CD} \pm 10 \%(\mathrm{~nm})$ & $>500$ & $>300$ & 100 & 125 \\
\hline \multicolumn{5}{|l|}{ Cross-section image } \\
\hline $\mathrm{CD}(\mathrm{nm})$ & 171.1 & 99.0 & 49.2 & 50.4 \\
\hline
\end{tabular}

Fig.13. Exposure tool compatibility of Lithographic property. 
crosslinking BARC and NCA42219. Control sample is conventional gap filling BARC with crosslinker. The new self-crosslinking BARC showed 3 times higher SC-1 resistance than that of control sample. Moreover, NCA42219 showed 16 times higher SC-1 resistance than that of control sample (Fig. 11). Since it shows very high SC-1 resistance, $\mathrm{SC}-1$ solution can be changed from weak to strong condition. Therefore, it is expected that the throughput is greatly improved.

\subsubsection{Etching rate}

Etch selectivity of NCA42219 was investigated to be calculated against that of ArF resist. Figure 12 was shown the Etch selectivity of BARC using $\mathrm{N}_{2}$ gas. New BARCs attained over 1.7 times faster against the resist. These results mean that NCA42219 can be used in various exposure process and improve the process throughput.

\subsubsection{Lithography}

Finally, NCA42219 functions as BARC in various exposure processes because it shows absorbance of both $\mathrm{ArF}$ and $\mathrm{KrF}$. We evaluated the litho performance with various exposure tools to check the exposure tool compatibility with difference $\mathrm{CD}$ size $(170 \mathrm{~nm}, 100 \mathrm{~nm}$, and $50 \mathrm{~nm})$. NCA42219 showed good litho performance that demonstrates good compatibility with various exposure tool and wide process margin (Fig. 13).

\section{Conclusion}

The BARC was investigated for improving gap filling property and $\mathrm{SC}-1$ resistance. In order to improve gap filling property, new self-crosslinking system has been investigated. New self-crosslinking system was needed high-activated catalyst and high temperature bake process. We found the key catalyst which enables this crosslinking reaction in low temperature baking process $\left(<215^{\circ} \mathrm{C}\right)$. And the new BARC with self-cross linking system showed good gap filling property in narrow trench pattern $(<10$ $\mathrm{nm})$.

On the other hand, in order to improve SC-1 resistance, new additive with high adhesion property to TiN has been investigated. In the screening, we found one characteristic additive which showed high adhesion to TiN surface. And it was proved that this additive was coordinated on TiN surface, especially by through the bake process. NCA42219 is one of the candidate BARC for implant and HKMG process to use self-crosslinking system and new additive, this material showed high gap filling property, high SC-1 resistance and more conventional required properties. Moreover, this BARC functioned as BARC in various exposure processes because it showed absorbance of both $\mathrm{KrF}$ and $\mathrm{ArF}$.

\section{References}

1. C. Y. Chang, D. C. Yu, J. H. Chen, J. C. H. Lin, B. J. Lin, J. W. Thackeray, V. Vohra, G. Wayton, and T. Kurihara, Proc. SPIE, 6153 (2006) 61530M.

2. Y. Hiroi, T. Kishioka, R. Sakamoto, D. Maruyama, T. Ohashi, T. Ishida, S. Kimura, Y. Sakaida, and H. Watanabe, Proc. SPIE, 6519 (2007) 651928.

3. S. Takei, Y. Sakaida, and T. Shinjo, Proc. SPIE, 6519 (2007) 65192V.

4. K. Komura, Y. Hishiro, G. Wakamatsu, Y. Takimoto, T. Nagai, T. Kimura, Y. Yamaguchi, T. Shimokawa, G. Breyta, N. Arellano, S. Balakrishnan, L. D. Bozano, A. Sankaranarayanan, K. M. Bajjuri, D. P. Sanders, C. E. Larson, A. DeSilva, and M. Glodde, Proc. SPIE, 9051 (2014) 905115. 